\section{Commentary: The feng shui of LVAD implantation}

\author{
Matthias Loebe, MD, PhD, FCCP, FACC, and \\ Ali Ghodsizad, MD, PhD, FACC, FETCS
}

One of the Five Arts of Chinese Metaphysics addresses the invisible forces that bind the universe, earth, and humanity together. Feng shui harmonizes the individual with the surrounding environment using energy forces. One lesson of this teaching says that the vital force-the chi-flows in curved lines and becomes a negative and destructive force, if it hits resistance or has to flow as a straight force. ${ }^{1}$

Whereas for the ancient Chinese the vital forces were invisible but omnipresent, modern imaging techniques, such as high-resolution computed tomography, make flows and forces visible to the human eye. Now we can study the impact of surgical techniques, anastomotic angles, and pump settings on the immediate surrounding environment of the outflow graft. ${ }^{2}$ We and others have measured wall stress and flow dynamics in the ascending aorta and the aortic arch. One sensitive structure very close to the anastomotic side of the outflow graft anastomosis is the aortic valve. In this issue of the Journal, Kasinpila and colleagues ${ }^{3}$ ventured into measuring the impact of different anastomotic locations on the fate of the valvular apparatus.

Several years ago, the left ventricular assist device (LVAD) community focused a huge amount of attention on the development of aortic valve insufficiency (AI) after VAD implantation; close to 70 publications appeared on this subject between 2013 and 2017. Then, in 2018, Truby and colleagues ${ }^{4}$ analyzed the INTERMACS database and studied 10,603 patients after LVAD implantation, and found that $15 \%$ developed progressive AI over a time frame of 2 years. Some required later interventions. Fine-tuning of systemic blood pressure and pump speed appeared to be beneficial and sufficient for managing these patients. After this landmark publication, very little attention has been

From the Division of Thoracic Transplantation and Mechanical Support, Miami Transplant Institute, Miami, Fla.

Disclosures: The authors reported no conflicts of interest.

The Journal policy requires editors and reviewers to disclose conflicts of interest and to decline handling or reviewing manuscripts for which they may have a conflict of interest. The editors and reviewers of this article have no conflicts of interest.

Received for publication April 16, 2020; revisions received April 16, 2020; accepted for publication April 17, 2020; available ahead of print May 13, 2020.

Address for reprints: Matthias Loebe, MD, PhD, FCCP, FACC, Thoracic Transplantation and Mechanical Support, Miami Transplant Institute, Miami, FL (E-mail: Mx11039@med.miami.edu).

J Thorac Cardiovasc Surg 2021;162:1564-6

$0022-5223 / \$ 36.00$

Copyright (c) 2020 by The American Association for Thoracic Surgery

https://doi.org/10.1016/j.jtcvs.2020.04.122

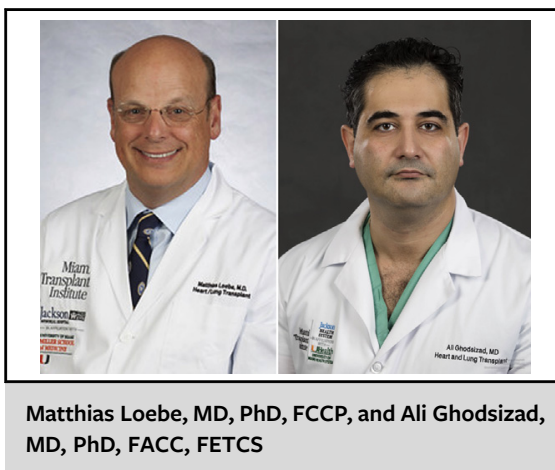

CENTRAL MESSAGE

Based on 3D reconstruction and

flow calculation, the authors

show that outflow graft position

and anastomotic technique alter

wall stress in aortic root and

contribute to the risk of aortic

insufficiency.

given to AI after LVAD. PubMed lists only 12 articles in the last 3 years. This does not necessarily mean that the problem has gone away, but it appears to have been less of an active issue in these patients than what some of us thought almost a decade ago.

Kasinpila and colleagues ${ }^{3}$ now present their findings in patients undergoing LVAD implantation studied with flow dynamic imaging. From computed tomography scans obtained after LVAD implantation, 3D models were created, and the geometry of the LVAD outflow graft in relation to the ascending aorta was quantified. These models were then used to run computational fluid dynamic simulations that calculated the pressure and wall shear stress exerted on the aortic root. A total of 10 patients with new onset of AI were compared with 20 patients who did not develop valve leakage. The authors found that the distance between aortic root and outflow graft anastomosis was shorter in patients who subsequently developed AI. Higher wall stress in the aortic root led to damage of the aortic valve.

This very elegant model provides impressive insight into the importance of surgical technique. Several authors have stressed that the surgical technique in performing the anastomosis to the ascending aorta has a significant impact on the sheer stress on the surface of the aortic valve. Iizuka and colleagues ${ }^{5}$ showed in their animal studies that the anastomotic angle determines the amount of stress to the valve leaflets (and the coronary flow as well). We have 
frequently mentioned that a 90-degree angle of the graft to the ascending aorta will produce high flows hitting the fragile leaflets of the aortic valve. We believe that this is particularly true when implanting the LVAD in the classic technique used by some, where the anastomosis to the aorta is done first and then the device is implanted into the apex. The outflow graft ends up at an almost 90-degree angle to the ascending aorta, and flow may be directed, at least in part, against the aortic valve. Leaving the graft long may change the angle at the anastomotic side.

Furthermore, alternative techniques, such as bringing the outflow graft up from the left margin and crossing the pulmonary artery, obviously have an impact on flow dynamics at the anastomotic site and in the aortic root. Attaching the graft to the descending aorta is another technique for implanting contemporary devices, particularly if the aim is to avoid the need for redo sternotomy. ${ }^{6}$ This outflow graft position used to be associated with prolonged low flow in the aortic root and a substantial risk of clot formation due to stasis at the valve. At least in our own experience, this problem seems to have disappeared with the advent of centrifugal pumps. We believe that the constant changes in flow observed in these pumps and their sensitivity to afterload have beneficial effects on washing out the aortic root when the outflow graft is attached to the descending aorta.

Even with anastomosing the outflow graft to the ascending aorta, there might be a difference between axial flow devices and centrifugal flow pumps, with the latter showing a much more pronounced variation in flow and thus in piston power. ${ }^{7}$ However, in their huge cohort of INTERMACS patients, Truby and colleagues ${ }^{4}$ did not see a significant difference based on pump design. Further computational flow dynamic studies may be of interest to further investigate this question.

Other reasons for the development of AI after LVAD implantation should be considered. In our experience, aortic valve damage may be caused by prolonged periods of Impella support with the pump sitting in the aortic valve. This may lead to some structural damage that then forms the basis of subsequent progression and significant valvular insufficiency.

The present era of mechanical support of the left ventricle started with the first human implantation of an axial flow pump in $1998 .{ }^{8}$ These miniaturized pumps only came into being after the application of computer-based flow simulation during the design process. These simulations led to less shear stress inside the pump and better washout of the bearing, and also helped redirect the blood flow by incorporating flow straighteners at the outflow side of the pump. Hemolysis was reduced to a minimum, and energy consumption was optimized. ${ }^{9}$ However, these studies were dedicated exclusively to optimizing the design of the pump itself. Little or no attention was given to the flow dynamics in the grafts and tubes leading into and out of the pump. It should come as no surprise that the principles that led to dramatic improvements in pump performance and pump safety also apply to the components downstream and those leading into the pump. As the work by Kasinpila and colleagues ${ }^{3}$ elegantly shows, a closer look at the outflow graft geometry is warranted to better understand some of the issues arising after LVAD implantation. This study adds to the knowledge accumulated over many years on the importance of flow dynamics and flow patterns in blood pumps. The findings should help us optimize our technique, improving immediate outcomes and preventing mid- and long-term damage to the environment in which the pump works.

When the ancient Chinese developed their philosophy of feng shui, when today's architects embrace that millionyear-old concept, the goal is to let the spiritual forces flow unimpeded, to create harmony and balance. Human blood seems to like being treated based on those concepts: if we interfere with the harmony and balance, coagulation and inflammation are stimulated and can wreak havoc. The vascular system and heart valves seem to dislike unbalanced forces as well. Harmonic flow patterns help maintain the balance and integrity of all these structures. We should improve our surgical technique accordingly. If this sounds esoteric to you, just look at pictures and findings in this article to see the good and negative forces at work, now in color and well visible to the naked eye. I hope that in the future we will be able to plan implantation based on computational models, and that we will find ways to immediately review the quality of our anastomosis by imaging techniques, avoiding damage and unnecessary stress to the surrounding structures. The pumps will then be able to operate in total harmony with the patient's body. Wouldn't that be great?

\section{References}

1. Walters D. The Feng Shui Handbook: A Practical Guide to Chinese Geomancy Hammersmith, London: Aquarian Press; 1991.

2. Karmonik C, Partovi S, Loebe M, Schmack B, Weymann A, Lumsden AB, et al Computational fluid dynamics in patients with continuous-flow left ventricular assist device support show hemodynamic alterations in the ascending aorta. $J$ Thorac Cardiovasc Surg. 2014;147:1326-33.e1.

3. Kasinpila P, Kong S, Fong R, Shad R, Kaiser A, Marsden A, et al. Use of patientspecific computational models for optimization of aortic insufficiency after implantation of left ventricular assist device. J Thorac Cardiovasc Surg. 2021;162: 1556-63.

4. Truby LK, Garan AR, Givens RC, Wayda B, Takeda K, Yuzefpolskaya M, et al. Aortic insufficiency during contemporary left ventricular assist device support: analysis of the INTERMACS registry. JACC Heart Fail. 2018;6:951-60.

5. Iizuka K, Nishinaka T, Akiyama D, Sumikura H, Mizuno T, Tsukiya T, et al. The angle of the outflow graft to the aorta can affect recirculation due to aortic insufficiency under left ventricular assist device support. J Artif Organs. 2018;21: 399-404.

6. Maltais S, Anwer LA, Tchantchaleishvili V, Haglund NA, Dunlay SM, Aaronson KD, et al. Left lateral thoracotomy for centrifugal continuous-flow left ventricular assist device placement: an analysis from the Mechanical Circulatory Support Research Network. ASAIO J. 2018;64:715-20.

7. Heatley G, Sood P, Goldstein D, Uriel N, Cleveland J, Middlebrook D, et al. Clinical trial design and rationale of the Multicenter Study of MagLev Technology in Patients Undergoing Mechanical Circulatory Support Therapy With HeartMate 3 (MOMENTUM 3) investigational device exemption clinical study protocol. $J$ Heart Lung Transpl. 2016;35:528-36. 
8. Potapov EV, Loebe M, Nasseri BA, Sinawski H, Koster A, Kuppe H, et al. Pulsatile flow in patients with a novel nonpulsatile implantable ventricular assist device. Circulation. 2000;102(19 Suppl 3):III183-7.
9. Wernicke JT, Meier D, Mizuguchi K, Damm G, Aber G, Benkowski R, et al. A fluid dynamic analysis using flow visualization of the Baylor/NASA implantable axial flow blood pump for design improvement. Artif Organs. 1995;19:161-77.
See Article page 1556.

\section{Commentary: Transcending acceptable, moving toward optimal: Standardizing surgical configurations of ventricular assist device therapy}

\author{
Song Li, MD, ${ }^{\mathrm{a}}$ Daniel Zimpfer, MD, ${ }^{\mathrm{b}}$ and
} Claudius Mahr, DO

The number of patients with end-stage heart failure potentially eligible for ventricular assist device (VAD) therapy continues to increase. ${ }^{1}$ Meanwhile, VAD outcomes with current centrifugal devices have improved significantly and in the first few years after implantation are approaching those of transplantation, yet at the same time they remain encumbered by adverse events such as stroke and hemodynamic complications like aortic regurgitation (AR). ${ }^{2}$ Our understanding of the mechanisms of VAD complications has expanded from basic engineering of the devices themselves to the patient-pump interface (comorbidities, thrombogenicity), medical management (blood pressure control, aortic valve opening), and surgical configuration. ${ }^{3,4}$ Computational fluid dynamic modeling (CFD) has emerged as a powerful method to investigate hemodynamics and thrombogenicity of various VAD surgical configurations. ${ }^{5-8}$

In this issue of the Journal, Kasinpila and colleagues ${ }^{9}$ examined how outflow graft position might contribute to de

From the a Division of Cardiology, Department of Medicine, University of Washington, Seattle, Wash; and ${ }^{b}$ Department of Cardiac Surgery, Medical University of Vienna, Vienna, Austria.

Disclosures: Dr Zimpfer is an investigator/consultant for Abbott and Medtronic. Dr Mahr is an investigator/consultant for Abbott, Medtronic, and Abiomed. Dr. Li reported no conflicts of interest.

The Journal policy requires editors and reviewers to disclose conflicts of interest and to decline handling or reviewing manuscripts for which they may have a conflict of interest. The editors and reviewers of this article have no conflicts of interest.

Received for publication April 30, 2020; revisions received April 30, 2020; accepted for publication May 4, 2020; available ahead of print May 15, 2020.

Address for reprints: Claudius Mahr, DO, Division of Cardiology, University of Washington, Seattle, WA 98195 (E-mail: cmahr@uw.edu).

J Thorac Cardiovasc Surg 2021;162:1566-7

$0022-5223 / \$ 36.00$

Copyright (c) 2020 by The American Association for Thoracic Surgery

https://doi.org/10.1016/j.jtcvs.2020.05.017

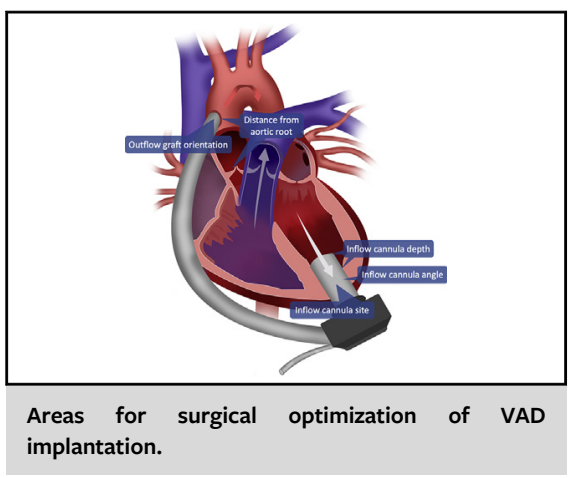

CENTRAL MESSAGE

An emerging body of evidence

supports that VAD surgical

configuration may contribute to

complications. Standardization

of optimal VAD surgical configu-

ration is imperative to improve

outcomes.

novo AR using a CFD model. Via 3-dimensional reconstructions of computed tomography-derived aortic geometry of $10 \mathrm{AR}$ and 20 non-AR VAD patients, the authors found that the distance from aortic root to outflow graft was significantly smaller in patients who developed AR, whereas outflow graft angles relative to the aorta did not appear to have a significant impact. CFD modeling also revealed larger recirculation zones and greater wall shear stress in the aortic root of patients with AR. Taken together, the current data establish a plausible theory that the surgical outflow graft configuration may, via hemodynamic alterations in the aortic root, contribute to development of AR during VAD support.

The authors' application of CFD evaluating the association of surgical configuration on the development of AR on support is highly pertinent, as AR is a common complication of continuous flow. ${ }^{10}$ Although the study benefits from a robust sample size and thoughtful analysis, its results need to be interpreted in the context of limitations inherent 